\title{
Low-fat yogurt consumption reduces biomarkers of chronic inflammation and inhibits markers of endotoxin exposure in healthy premenopausal women: a randomised controlled trial
}

\author{
Ruisong Pei ${ }^{1,2}$, Diana M. DiMarco ${ }^{1}$, Kelley K. Putt ${ }^{2}$, Derek A. Martin ${ }^{1,2}$, Qinlei Gu ${ }^{2}$, \\ Chureeporn Chitchumroonchokchai ${ }^{3}$, Heather M. White ${ }^{4}$, Cameron O. Scarlett ${ }^{5}$, \\ Richard S. Bruno ${ }^{3}$ and Bradley W. Bolling ${ }^{1,2 *}$ \\ ${ }^{1}$ Department of Nutritional Sciences, University of Connecticut, 3624 Horsebarn Road Extension, Unit 4017, Storrs, CT 06269, USA \\ ${ }^{2}$ Department of Food Science, University of Wisconsin-Madison, 1605 Linden Drive, Madison, WI 53706, USA \\ ${ }^{3}$ Human Nutrition Program, The Obio State University, 1787 Neil Avenue, Columbus, OH 43210, USA \\ ${ }^{4}$ Department of Dairy Science, University of Wisconsin-Madison, 1675 Observatory Drive, Madison, WI 53706, USA \\ ${ }^{5}$ School of Pharmacy, University of Wisconsin-Madison, 777 Highland Avenue, Madison, WI 53705, USA \\ (Submitted 1 September 2017 - Final revision received 5 October 2017 - Accepted 6 October 2017- First published online 28 November 2017)
}

\section{Abstract}

The anti-inflammatory mechanisms of low-fat dairy product consumption are largely unknown. The objective of this study was to determine whether low-fat yogurt reduces biomarkers of chronic inflammation and endotoxin exposure in women. Premenopausal women (BMI 18.5-27 and $30-40 \mathrm{~kg} / \mathrm{m}^{2}$ ) were randomised to consume $339 \mathrm{~g}$ of low-fat yogurt (yogurt non-obese (YN); yogurt obese (YO)) or 324 g of soya pudding (control non-obese; control obese (CO)) daily for 9 weeks ( $n$ 30/group). Fasting blood samples were analysed for IL-6, TNF- $\alpha$ /soluble TNF II (sTNF-RII), high-sensitivity C-reactive protein, 2-arachidonoyl glycerol, anandamide, monocyte gene expression, soluble CD14 (sCD14), lipopolysaccharide (LPS), LPS binding protein (LBP), IgM endotoxin-core antibody (IgM EndoCAb), and zonulin. BMI, waist circumference and blood pressure were also determined. After 9-week yogurt consumption, YO and YN had decreased TNF- $\alpha$ /sTNFR-RII. Yogurt consumption increased plasma IgM EndoCAb regardless of obesity status. SCD14 was not affected by diet, but LBP/sCD14 was lowered by yogurt consumption in both YN and YO. Yogurt intervention increased plasma 2-arachidonoylglycerol in YO but not YN. YO peripheral blood mononuclear cells expression of NF- $\kappa \mathrm{B}$ inhibitor $\alpha$ and transforming growth factor $\beta 1$ increased relative to CO at 9 weeks. Other biomarkers were unchanged by diet. CO and YO gained approximately $0.9 \mathrm{~kg}$ in body weight. YO had 3.6\% lower diastolic blood pressure at week 3 . Low-fat yogurt for 9 weeks reduced biomarkers of chronic inflammation and endotoxin exposure in premenopausal women compared with a non-dairy control food. This trial was registered as NCT01686204.

Key words: Obesity: Low-fat yogurt: Chronic inflammation: Endotoxin exposure: Dairy products

Obesity is associated with increased risk of CVD, type 2 diabetes, hypertension and certain cancers $^{(1)}$. These comorbidities are associated with chronic inflammation ${ }^{(1)}$. Unresolved, low-grade, obesity-associated inflammation originates from the interplay between immune cells and metabolic tissues such as adipose, liver, muscle, pancreas and brain in response to excessive nutrient intake, leading to increased proinflammatory cytokine release ${ }^{(2)}$. Inflammation is exacerbated by chronic endotoxin exposure resulting from compromised intestinal barrier function in obese individuals ${ }^{(3,4)}$. Endotoxin, especially the Gram-negative-derived lipopolysaccharide (LPS) from gut microbiota, induces inflammation in humans ${ }^{(5)}$. LPS inflammatory signaling is mediated by its translocation by lipopolysaccharide-binding protein (LBP) and membranebound or soluble CD14 (sCD14) to Toll-like receptor 4 (TLR4)/MD2 complex ${ }^{(6)}$. The endocannabinoid (eCB) system also modulates intestinal barrier function and metabolic endotoxaemia associated with obesity ${ }^{(4)}$.

Yogurt consumption appears to be a promising strategy to improve obesity-associated intestinal barrier dysfunction and prevent chronic inflammation. Consumption of Lactobacilluscontaining yogurt reduced surrogate markers of endotoxaemia and decreased intestinal permeability in individuals with compromised gut barrier integrity ${ }^{(7,8)}$. Smaller intervention studies

Abbreviations: 2-AG, 2-arachidonoylglycerol; AEA, anandamide; CO, control obese; IgM EndoCAb, IgM endotoxin-core antibody; LBP, lipopolysaccharidebinding protein; LPS, lipopolysaccharide; sCD14, soluble CD14; TLR4, Toll-like receptor 4; YN, yogurt non-obese; YO, yogurt obese.

* Corresponding author: B. W. Bolling, fax +1 608262 6872, email bwbolling@wisc.edu 
demonstrated that yogurt consumption reduced biomarkers of inflammation in elderly individuals and children ${ }^{(9,10)}$. Epidemiological studies have also associated increased dairy product consumption with decreased risk of chronic diseases such as CVD, type 2 diabetes and hypertension ${ }^{(11-13)}$. However, the effect of yogurt consumption on inflammation and endotoxin exposure in healthy premenopausal women is still largely unknown. We hypothesised that regular consumption of a commercial low-fat yogurt would reduce biomarkers of chronic inflammation and endotoxin exposure in healthy premenopausal women. We tested this hypothesis by conducting a randomised trial using a macronutrient- and texture-matched non-dairy food as a control.

\section{Methods}

\section{Chemicals and reagents}

Anandamide (AEA), 2-arachidonoylglycerol (2-AG), anandamide-d4 (D4-AEA) and 2-arachidonoyl glycerol-d5 were purchased from Cayman Chemical. Acetonitrile, formic acid, acetic acid, Optima ${ }^{\mathrm{TM}}$ LC/MS grade water, isopropanol, ethanol and chloroform were purchased from Fisher Scientific. Histopaque $^{\circledR}-1077$ was purchased from Sigma-Aldrich. PBS was purchased from MP Biomedicals. RNAlater and TRIzol reagent were purchased from Life Technologies. RNase-free water was purchased from Dot Scientific. RNase-free DNase was purchased from Qiagen.

\section{Participants}

This study was conducted according to the guidelines laid down in the Declaration of Helsinki and all procedures involving human participants were approved by the Institutional Review Boards at the University of Connecticut (no. H12-168) and University of Wisconsin-Madison (no. 2014-0669). Written informed consent was obtained from all participants. This trial was registered at clinicaltrials.gov as NCT01686204. The study inclusion criteria included: BMI from 18.5 to $27 \mathrm{~kg} / \mathrm{m}^{2}$ or from 30 to $40 \mathrm{~kg} / \mathrm{m}^{2}$, age 21 to 55 years, stable body weight for the previous 2 months, willing to avoid yogurt and probioticcontaining foods and consume the provided $339 \mathrm{~g}$ of yogurt or $324 \mathrm{~g}$ soya pudding (control treatment) for the duration of the study, no previous diagnosis of CVD, diabetes or arthritis, not being currently treated for cancer, not taking any antiinflammatory drugs or prescribed oestrogen replacement therapy, not on slimming, vegetarian or vegan diets, not currently taking dietary supplements or smoking, not allergic to soya, egg or milk, not pregnant, lactating or seeking to become pregnant.

\section{Dietary treatment}

The dietary intervention consisted of commercially available low-fat yogurt (Yoplait, General Mills, Inc.), and soya pudding (unfermented) as a macronutrient and micronutrient, energy content and texture-matched control food (ZenSoy). The nutrients provided daily by the two test foods are described in Table 1.
Table 1. Nutrient composition of daily consumption of low-fat yogurt and non-dairy product control snack.

\begin{tabular}{lcc}
\hline Nutrients & Low-fat yogurt (Yoplait) & Control food (ZenSoy) $\dagger$ \\
\hline Serving (g) & 339 & 324 \\
Energy content (kJ) & 1381 & 1381 \\
Energy content (kcal) & 330 & 330 \\
Total fat (g) & 3 & 3 \\
Carbohydrate (g) & 66 & 66 \\
Protein (g) & 9 & $6-9 \ddagger$ \\
Cholesterol (mg) & 15 & 0 \\
Na (mg) & 180 & $165-210 \S$ \\
Ca (mg) & 450 & $180-450 \S$ \\
Vitamin A (IU) & 1500 & 600 \\
Vitamin D ( $\mu \mathrm{g})$ & 6.75 & 6.75 \\
\hline
\end{tabular}

* Ingredients included: cultured pasteurised grade A low-fat milk, sugar, strawberries (or banana purée/peaches/raspberries, depending on the flavours), modified maize starch, non-fat milk, Kosher gelatin, citric acid, tricalcium phosphate, coloured with carmine, natural flavour, pectin, vitamin $A$ acetate, vitamin $D_{3}$. Contained disclaimer that product meets National Yogurt Association criteria for live and active culture yogurt (Contains Lactobacillus bulgaricus and Streptococcus thermophilus with at least $10^{7}$ cultures $/ \mathrm{g}$ at manufacture).

$\dagger$ Ingredients included: filtered water, organic soyamilk, organic granulated cane sugar, organic maize starch, organic cocoa (processed with alkali) or organic vanilla extract, natural flavours, carrageenan, calcium carbonate, evaporated salt, organic soya lecithin, organic locust bean gum, vitamin $A$ palmitate, vitamin $D_{2}$, riboflavin, vitamin $B_{12}$.

‡ Different flavours of control snack contained either 6 or $9 \mathrm{~g}$ of protein.

$\S$ Due to the reformulation by the manufacturer during the intervention, the sodium content decreased from 210 to $165 \mathrm{mg}$; the calcium decreased from 450 to $180 \mathrm{mg}$ ( $n$ 24, on the old formulation; $n$ 36, on the new formulation).

\section{Experimental design}

We performed a randomised, controlled study of parallel design to examine how yogurt consumption affects chronic inflammation and intestinal barrier function relative to the consumption of a non-dairy control food. sCD14 has been used as a biomarker for endotoxin exposure and was thus chosen as the primary outcome $^{(14)}$. For the sample size calculation, an $8.2 \%$ margin of error for sCD14 was determined for a moderate group size ( $n$ 30/group) based on the standard deviation of $\mathrm{SCD} 14$ in a prior study ${ }^{(14)}$. Apparently healthy premenopausal women were recruited from the Storrs, CT and Madison, WI area from October 2012 to April 2015. After the initial screening, 128 subjects were enrolled and randomly assigned to either the yogurt group or the soya pudding control group, in blocks of six ${ }^{(15)}$. Participants were randomised upon enrolment by assigning random numbers generated by Minitab 17.0 (Minitab Inc.). To avoid confounding by probiotic or dairy product consumption, the participants were instructed to restrict consumption of dietary supplements, fermented foods and limit their dairy product consumption to $\leq 4$ servings/d for 2 weeks before the intervention (washout period, weeks -2-0) and throughout the intervention. From the beginning of week 0 to the end of week 9 (intervention period), the participants consumed $339 \mathrm{~g}$ of yogurt $(12 \mathrm{oz}$.) or $324 \mathrm{~g}$ of control food daily. The lids of consumed yogurt and pudding cups were collected weekly to assess compliance. Participants visited the study centre at weeks 0 , 3, 6 and 9 for anthropometric and blood pressure measurements. In addition, fasting blood samples were collected from the antecubital vein into evacuated tubes containing EDTA or sodium heparin (Becton, Dickinson and Company). At weeks 0 and 9, peripheral blood mononuclear cells (PBMC) were immediately isolated from $20 \mathrm{ml}$ of sodium heparin blood samples for later mRNA analysis. For biomarker analysis, plasma was prepared 
from blood samples held on ice and centrifuged $\left(4^{\circ} \mathrm{C}, 15 \mathrm{~min}\right.$, $1500 \mathrm{~g}$ ) within $20 \mathrm{~min}$ of collection. Aliquots of plasma were transferred to sterile cryogenic vials and snap-frozen in liquid $\mathrm{N}_{2}$. All samples were stored at $-80^{\circ} \mathrm{C}$ until analysis.

\section{Anthropometric and blood pressure measurements}

Body weight and height were measured on a digital physician scale to the nearest $0.1 \mathrm{~kg}$ and $1 \mathrm{~cm}$ (H150-10-5; Rice Lake Weighing Systems). Waist circumference was determined by placing a measuring tape evenly around a bare abdomen at the iliac crest. Blood pressure was measured using an Omron HEM-780 with ComFit cuff for non-obese participants, or an Omron BP710 with Omron H003D large adult cuff for obese participants (Omron Healthcare, Inc.). Before readings, participants were instructed to sit upright in a phlebotomy chair for a minimum of $5 \mathrm{~min}$ with their back supported, feet on the floor, legs uncrossed, bladder empty, and their upper arm supported at heart level ${ }^{(16)}$. Blood pressure was measured twice, at least $3 \mathrm{~min}$ apart. If readings were more than $5 \mathrm{mmHg}$ apart, a third reading was taken.

\section{Inflammatory biomarkers}

IL- 6 and TNF- $\alpha$ in EDTA plasma were measured by human highsensitivity ELISA kits (IL-6, catalogue no. SS600B; TNF- $\alpha$, catalogue no. SSTA00D; R\&D System). Soluble TNF-Receptor II (sTNF-RII) was measured by a human ELISA kit (catalogue no. SRT200; R\&D System). High-sensitivity C-reactive protein (hsCRP) was measured by a human ELISA kit (catalogue no. BC-1119; BioCheck Inc.). All the measurements were performed on a SpectraMax M2 microplate reader (Molecular Devices), following the manufacturer's instruction.

\section{Biomarkers of endotoxin exposure and intestinal barrier function}

LBP and IgM endotoxin-core antibody (IgM EndoCAb) in EDTA plasma were measured by human ELISA kits (LBP, catalogue no. HK315; IgM EndoCAb, catalogue no. HK504-IGM; Hycult Biotech). EDTA plasma LPS was measured by the PyroGene Recombinant Factor C endotoxin Detection System (catalogue no. 50-658U; Lonza Group Ltd). EDTA plasma zonulin was measured by a human ELISA kit (catalogue no. K5600; Immundiagnostik AG). Sodium heparin plasma sCD14 was measured by a human ELISA kit (catalogue no. DC140; R\&D System). All the measurements were performed on a SpectraMax M2 microplate reader (Molecular Devices), following the manufacturers' instruction. The ratio between LBP and SCD14 was calculated as a marker for endotoxaemia. Plasma AEA and 2-AG were quantitated by UPLCMS/MS after protein precipitation and cleanup by Ostro passthrough sample preparation plate. These methods are described further in the online Supplementary material.

\section{Peripheral blood mononuclear cells isolation and mRNA analysis}

PBMC were isolated from sodium heparin blood by gradient centrifugation using Histopaque ${ }^{\circledR}-1077$ and stored at $-80^{\circ} \mathrm{C}$. RNA was extracted from PMBC by TRIzol reagent and further purified with an RNeasy mini kit (Qiagen). After subsequent complementary DNA synthesis, RT quantitative PCR was performed using the $\mathrm{iTaq}^{\mathrm{TM}}$ Universal $\mathrm{SYBR}^{\circledR}$ Green Supermix (Bio-Rad), on a Bio-Rad CFX96 system (Bio-Rad). A detailed description of the methodology and primer sequences are in the online Supplementary material and Table S1.

\section{Dietary analysis}

Participants were instructed to maintain their usual dietary pattern and energy intake, accounting for the additional $1255 \mathrm{~kJ}$ $(330 \mathrm{kcal})$ provided by the intervention foods. Dietary records were used to examine if the intervention changed participants' nutrients intakes or dietary patterns and to determine their compliance to the requested dietary restrictions. Participants were instructed to complete 3 -d food records on two nonconsecutive weekdays and 1 weekend day of their choice during the washout period and near the end of the intervention. Non-consecutive days were used to include a more representative record of foods in the dietary records ${ }^{(17)}$. Participants were given detailed instructions on how to complete the record by study staff. The food records were reviewed by study staff upon submission and any incomplete or inaccurate information was corrected with participant input. The self-reported dietary intakes were analysed by 2013 Nutrition Data System for Research (NCC).

\section{Statistical analysis}

All results were expressed as mean values with their standard errors. Statistical analysis was conducted on SAS 9.4 software. The significance level was set at $\alpha=0.05$ for all tests. At baseline, the differences of obese $v$. non-obese were analysed by independent $t$ test (PROC TTEST). Pearson's correlation coefficients of pairwise variables were determined by linear regression modelling (PROC CORR). The effects of dietary treatment (yogurt $v$. control), obesity (obese $v$. non-obese) and treatment $\times$ obesity on BMI, waist circumference and blood pressure were determined by two-way repeated-measures ANOVA with time as a covariate (PROC MIXED). At week 9, the difference in biomarkers between yogurt and control treatment groups was analysed by ANCOVA with baseline as the covariate (PROC GLM). For data analysis that required normal distribution, the Shapiro-Wilk test (PROC UNIVARIATE) was used for testing normality. If data failed the normality test, logtransformation was performed to achieve normality.

\section{Results}

In brief, 128 subjects were recruited. Eight subjects were excluded or dropped from the study due to personal or compliance issues. At the end of the study, sixty obese $(30-40 \mathrm{~kg} /$ $\mathrm{m}^{2}$ ) and sixty non-obese $\left(18.5-27 \mathrm{~kg} / \mathrm{m}^{2}\right)$ participants completed the study, with $n$ 30/group (yogurt obese (YO); control obese (CO); yogurt non-obese (YN); control non-obese) (Fig. 1). Due to the serving size, appearance and taste of intervention food, we were unable to blind participants. Nevertheless, all groups showed similar compliance of $>95 \%$. 


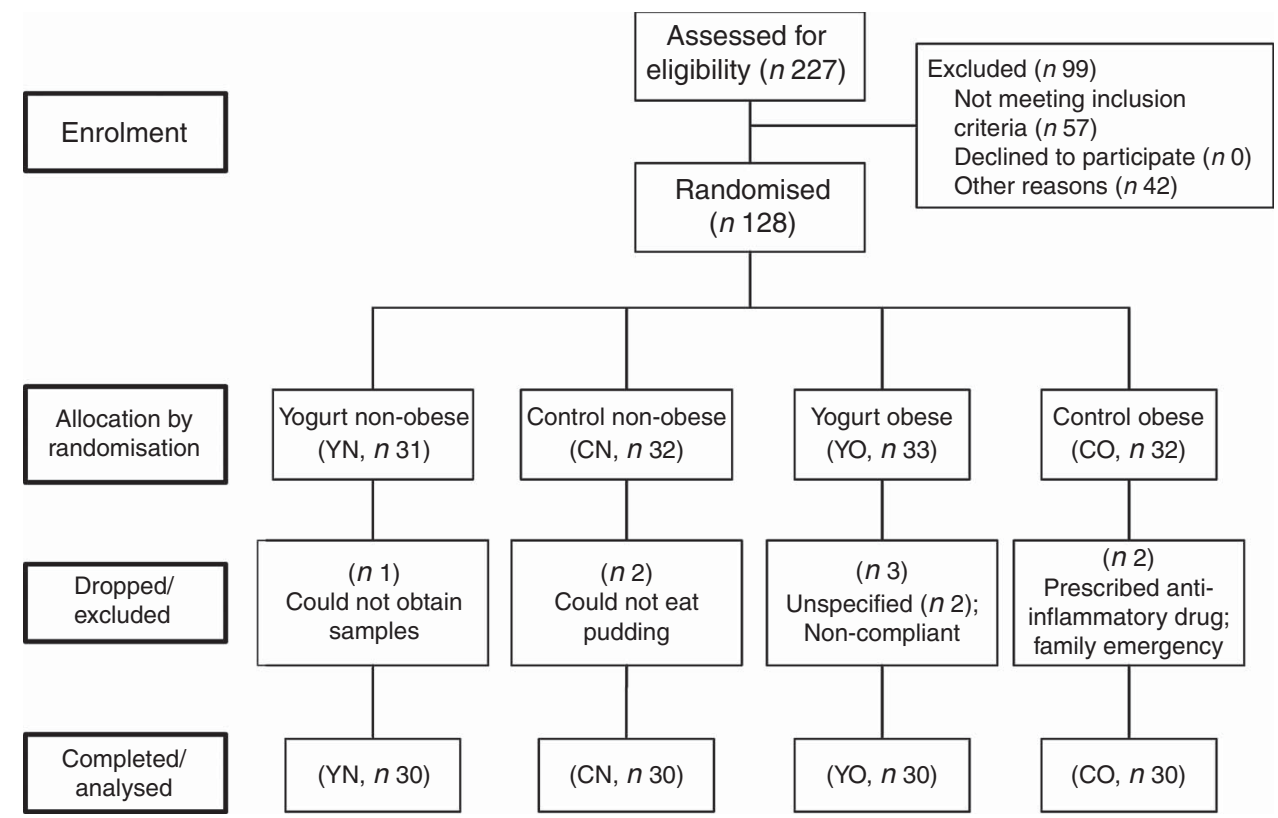

Fig. 1. Enrolment and follow-up of participants. $\mathrm{CN}$, control non-obese; $\mathrm{CO}$, control obese; YN, yogurt non-obese; YO, yogurt obese.

Table 2. Baseline anthropometric of enrolled participants

(Mean values with their standard errors; $n$ 30/group)

\begin{tabular}{|c|c|c|c|c|c|c|c|c|c|}
\hline \multirow[b]{3}{*}{ Characteristics } & \multicolumn{8}{|c|}{ Group } & \multirow{3}{*}{$\frac{\text { Significance }(P)^{*}}{\text { Obese } v \text {. lean }}$} \\
\hline & \multicolumn{2}{|c|}{$\mathrm{CN}$} & \multicolumn{2}{|c|}{$\mathrm{CO}$} & \multicolumn{2}{|c|}{ YN } & \multicolumn{2}{|c|}{ YO } & \\
\hline & Mean & SE & Mean & SE & Mean & SE & Mean & SE & \\
\hline Age (years) & $25 \cdot 3$ & $1 \cdot 1$ & 31.9 & 1.6 & $24 \cdot 8$ & 0.8 & $36 \cdot 7$ & $2 \cdot 0$ & $<0.0001$ \\
\hline Weight (kg) & $62 \cdot 3$ & 1.6 & 93.5 & $2 \cdot 2$ & $64 \cdot 7$ & 1.5 & 94.1 & 1.9 & $<0.0001$ \\
\hline Height $(m)$ & 1.66 & 0.01 & 1.65 & 0.01 & 1.67 & 0.01 & 1.65 & 0.01 & 0.2883 \\
\hline $\operatorname{BMI}\left(\mathrm{kg} / \mathrm{m}^{2}\right)$ & 22.5 & 0.4 & 34.3 & 0.5 & $23 \cdot 3$ & 0.5 & 34.4 & 0.7 & $<0.0001$ \\
\hline WC (cm) & 69.5 & 1.4 & $96 \cdot 3$ & 1.6 & 73.7 & 1.4 & 94.6 & 1.4 & $<0.0001$ \\
\hline SysBP (mmHg) & 105.5 & $2 \cdot 1$ & 116.9 & $2 \cdot 2$ & 103.5 & $2 \cdot 0$ & $116 \cdot 8$ & $2 \cdot 1$ & $<0.0001$ \\
\hline $\mathrm{DiaBP}(\mathrm{mmHg})$ & 73.3 & $1 \cdot 7$ & $79 \cdot 0$ & 1.5 & 71.3 & 1.5 & $79 \cdot 3$ & 1.3 & $<0.0001$ \\
\hline IL-6 (pg/ml) & 0.74 & 0.08 & 1.56 & 0.13 & 0.88 & 0.13 & 1.86 & 0.22 & $<0.0001$ \\
\hline hsCRP (mg/l) & $1 \cdot 24$ & 0.26 & 2.97 & 0.31 & $1 \cdot 15$ & 0.21 & $2 \cdot 63$ & 0.37 & $<0.0001$ \\
\hline TNF- $a(\mathrm{pg} / \mathrm{ml})$ & $1 \cdot 10$ & 0.07 & 1.25 & 0.10 & 1.14 & 0.09 & 1.52 & 0.12 & 0.0104 \\
\hline sTNF-RII (pg/ml) & 2125 & 72 & 2452 & 94 & 2028 & 69 & 2550 & 101 & $<0.0001$ \\
\hline LPS (EU/ml) & $14 \cdot 2$ & 0.9 & $16 \cdot 2$ & $1 \cdot 2$ & 11.9 & 1.0 & 13.8 & 0.8 & 0.2005 \\
\hline $\operatorname{LBP}(\mu \mathrm{g} / \mathrm{ml})$ & 9.9 & 0.9 & $12 \cdot 4$ & 0.7 & $9 \cdot 3$ & 0.6 & $12 \cdot 3$ & 0.9 & 0.0010 \\
\hline sCD14 (ng/ml) & 1421 & 50 & 1481 & 45 & 1402 & 59 & 1388 & 45 & 0.6579 \\
\hline LBP:sCD14 & $7 \cdot 14$ & 0.69 & 8.62 & 0.58 & $6 \cdot 89$ & 0.44 & $9 \cdot 13$ & 0.73 & 0.0032 \\
\hline IgM EndoCAb (MMU/ml) & 99.6 & $10 \cdot 6$ & $70 \cdot 7$ & $4 \cdot 2$ & $101 \cdot 4$ & $10 \cdot 1$ & 68.7 & $7 \cdot 1$ & 0.0004 \\
\hline $\mathrm{AEA}(\mathrm{nm})$ & 0.75 & 0.04 & $1 \cdot 15$ & 0.06 & 0.85 & 0.04 & $1 \cdot 10$ & 0.06 & $<0.0001$ \\
\hline 2-AG (nM) & 3.46 & 0.29 & 4.60 & 0.33 & 3.45 & 0.30 & $4 \cdot 10$ & 0.27 & 0.0033 \\
\hline
\end{tabular}

$\mathrm{CN}$, control non-obese; CO, control obese; YN, yogurt non-obese; YO, yogurt obese; WC, waist circumference; SysBP, systolic blood pressure; DiaBP, diastolic blood pressure; EU, endotoxin units; LPS, lipopolysaccharide; EU, endotoxin units; LBP, lipopolysaccharide-binding protein; sCD14, soluble CD14; IL-6, IL-6; hsCRP, high-sensitivity C-reactive protein; sTNF-RII, soluble TNF-receptor II; IgM EndoCAb, IgM endotoxin-core antibody; MU, median units; AEA, anandamide; 2-AG, 2-arachidonoylglycerol.

* Differences between obese and non-obese subjects were determined by independent $t$ test (PROC TTEST).

The baseline characteristics of participants are described in Table 2. The obese group was older and had higher blood pressure than the non-obese group $(P<0 \cdot 0001)$. Compared with non-obese participants, obese participants had higher IL-6, hsCRP, TNF- $\alpha$ indicating chronic inflammation, and had higher LBP, LBP:sCD14 ratio and lower IgM EndoCAb indicating increased endotoxin exposure $(P<0.05)$. Notably, baseline sCD14 and LPS were not different between non-obese and obese groups $(P=0.6579$ and $P=0.2005$, respectively). All measured biomarkers for inflammation and endotoxin exposure except for sCD14 and LPS were correlated with BMI (online Supplementary Table S2).

\section{Fasting plasma markers of inflammation}

Low-fat yogurt consumption for 9 weeks led to decreased TNF- $\alpha \quad\left(P_{\text {treatment }}=0.0219\right)$ and the TNF- $\alpha /$ sTNF-RII ratio $\left(P_{\text {treatment }}=0.0013\right)$ in both $\mathrm{YN}$ and $\mathrm{YO}$ (Table 3). Compared with baseline levels, YO had $7 \%$ decreased TNF- $\alpha /$ sTNF-RII ratio. The obesity status had no effect on TNF- $\alpha$ or the TNF- $\alpha /$ sTNF-RII ratio. 
Table 3. Changes in fasting plasma biomarkers of chronic inflammation and endotoxin exposure

(Mean values with their standard errors; $n$ 30/group)

\begin{tabular}{|c|c|c|c|c|c|c|c|c|}
\hline \multirow[b]{3}{*}{ Biomarkers } & \multirow[b]{3}{*}{ Group } & \multicolumn{4}{|c|}{ Time (weeks) } & & & \\
\hline & & \multicolumn{2}{|c|}{0} & \multicolumn{2}{|c|}{9} & \multicolumn{3}{|c|}{ Significance $(P)^{*}$} \\
\hline & & Mean & SE & Mean & SE & Treatment & Obesity & Treatment $\times$ obesity \\
\hline \multirow[t]{4}{*}{ IL-6 (pg/l) } & $\mathrm{CN}$ & 0.74 & 0.08 & 0.89 & 0.11 & 0.4408 & 0.3934 & 0.6610 \\
\hline & YN & 0.88 & 0.13 & 0.87 & 0.10 & & & \\
\hline & $\mathrm{CO}$ & 1.56 & 0.13 & 1.47 & 0.11 & & & \\
\hline & YO & 1.86 & 0.22 & 1.59 & 0.19 & & & \\
\hline \multirow{4}{*}{ hsCRP (mg/l) } & $\mathrm{CN}$ & 1.24 & 0.26 & 1.33 & 0.23 & 0.2670 & 0.5546 & 0.3711 \\
\hline & YN & 1.15 & 0.21 & 1.22 & 0.22 & & & \\
\hline & $\mathrm{CO}$ & 2.97 & 0.31 & 2.98 & 0.32 & & & \\
\hline & YO & 2.63 & 0.37 & 2.42 & 0.32 & & & \\
\hline \multirow[t]{4}{*}{ TNF-a/sTNF-RII (\%o) } & $\mathrm{CN}$ & 0.52 & 0.03 & 0.53 & 0.03 & 0.0013 & 0.9574 & 0.8901 \\
\hline & YN & 0.57 & 0.05 & 0.53 & 0.04 & & & \\
\hline & $\mathrm{CO}$ & 0.51 & 0.04 & 0.53 & 0.04 & & & \\
\hline & YO & 0.59 & 0.04 & 0.55 & 0.04 & & & \\
\hline \multirow[t]{4}{*}{ TNF- $a(\mathrm{pg} / \mathrm{ml})$} & $\mathrm{CN}$ & 1.10 & 0.07 & 1.21 & 0.09 & 0.0219 & 0.8384 & 0.0827 \\
\hline & YN & $1 \cdot 14$ & 0.09 & 1.05 & 0.09 & & & \\
\hline & $\mathrm{CO}$ & 1.25 & $0 \cdot 10$ & 1.23 & 0.08 & & & \\
\hline & YO & 1.52 & 0.12 & 1.42 & 0.10 & & & \\
\hline \multirow{4}{*}{ sTNF-RII (pg/ml) } & $\mathrm{CN}$ & 2130 & 70 & 2210 & 90 & 0.5178 & 0.5843 & 0.0108 \\
\hline & YN & 2030 & 70 & 2020 & 70 & & & \\
\hline & $\mathrm{CO}$ & 2450 & 90 & 2390 & 100 & & & \\
\hline & YO & 2550 & 100 & 2640 & 100 & & & \\
\hline \multirow[t]{4}{*}{ LBP/sCD14 ratio } & $\mathrm{CN}$ & $7 \cdot 14$ & 0.69 & 8.32 & 0.85 & 0.0477 & 0.0995 & 0.2227 \\
\hline & YN & $6 \cdot 89$ & 0.44 & 7.72 & 0.64 & & & \\
\hline & $\mathrm{CO}$ & 8.62 & 0.58 & 9.60 & 0.68 & & & \\
\hline & YO & $9 \cdot 13$ & 0.73 & $8 \cdot 71$ & 0.68 & & & \\
\hline \multirow[t]{4}{*}{ LBP $(\mu \mathrm{g} / \mathrm{ml})$} & $\mathrm{CN}$ & 9.9 & 0.9 & 10.5 & 1.0 & 0.1098 & 0.4572 & 0.6965 \\
\hline & YN & $9 \cdot 3$ & 0.6 & 9.4 & 0.6 & & & \\
\hline & $\mathrm{CO}$ & $12 \cdot 4$ & 0.7 & $12 \cdot 9$ & 0.9 & & & \\
\hline & YO & $12 \cdot 3$ & 0.9 & 11.7 & 0.8 & & & \\
\hline \multirow[t]{4}{*}{$\mathrm{sCD} 14(\mathrm{ng} / \mathrm{ml})$} & $\mathrm{CN}$ & 1421 & 50 & 1323 & 48 & 0.9977 & 0.2134 & 0.0755 \\
\hline & YN & 1402 & 59 & 1287 & 55 & & & \\
\hline & $\mathrm{CO}$ & 1481 & 45 & 1365 & 44 & & & \\
\hline & YO & 1388 & 45 & 1388 & 49 & & & \\
\hline \multirow[t]{4}{*}{ LPS (EU/ml) } & $\mathrm{CN}$ & $14 \cdot 2$ & 0.9 & $16 \cdot 6$ & 0.7 & 0.0548 & 0.0190 & 0.1962 \\
\hline & YN & 11.9 & 1.0 & $16 \cdot 2$ & 1.0 & & & \\
\hline & $\mathrm{CO}$ & $16 \cdot 2$ & 1.2 & 13.4 & 0.9 & & & \\
\hline & YO & 13.8 & 0.8 & $16 \cdot 7$ & 1.1 & & & \\
\hline \multirow[t]{4}{*}{ EndoCab IgM (MMU/ml) } & $\mathrm{CN}$ & 99.6 & $10 \cdot 6$ & 97.9 & 9.6 & 0.0052 & 0.4230 & 0.3939 \\
\hline & YN & 101 & 10 & 107 & 11 & & & \\
\hline & $\mathrm{CO}$ & 70.7 & 4.2 & 70.0 & 4.0 & & & \\
\hline & YO & 68.7 & $7 \cdot 1$ & $72 \cdot 2$ & 8.0 & & & \\
\hline \multirow[t]{4}{*}{ AEA (nм) } & $\mathrm{CN}$ & 0.75 & 0.04 & 0.78 & 0.05 & 0.5334 & 0.1620 & 0.1565 \\
\hline & YN & 0.85 & 0.04 & 0.80 & 0.05 & & & \\
\hline & $\mathrm{CO}$ & 1.15 & 0.06 & 1.03 & 0.06 & & & \\
\hline & YO & $1 \cdot 10$ & 0.06 & $1 \cdot 11$ & 0.08 & & & \\
\hline \multirow[t]{4}{*}{ 2-AG (nм) } & $\mathrm{CN}$ & 3.46 & 0.29 & 3.43 & 0.31 & 0.1188 & 0.7493 & 0.0372 \\
\hline & YN & 3.45 & 0.30 & 3.30 & 0.35 & & & \\
\hline & $\mathrm{CO}$ & 4.60 & 0.33 & $3 \cdot 74^{\mathrm{a}}$ & 0.32 & & & \\
\hline & YO & 4.10 & 0.27 & $4.11^{\mathrm{b}}$ & 0.30 & & & \\
\hline
\end{tabular}

CN, control non-obese; CO, control obese; YN, yogurt non-obese; YO, yogurt obese; hsCRP, high-sensitivity C-reactive protein; sTNF-RII, soluble TNF II; LPS, lipopolysaccharide; EU, endotoxin units; LBP, lipopolysaccharide-binding protein; sCD14, soluble CD14; IgM EndoCAb, IgM endotoxin-core antibody; MU, median units; AEA, anandamide; 2-AG, 2-arachidonoylglycerol.

a,b Mean values within a column with unlike superscript letters were significantly different $(P<0.05)$ by two-tailed $t$ test.

* The group difference at week 9 was compared by ANCOVA with baseline (week 0) as covariate (PROC GLM).

On the other hand, neither plasma IL-6 nor hsCRP was affected by dietary treatment or obesity status.

\section{Fasting biomarkers of endotoxin exposure and intestinal barrier function}

After 9 weeks intervention, IgM EndoCAb increased in both YN and YO $\left(P_{\text {treatment }}=0.0052\right.$ ) (Table 3$)$. The primary study outcome, fasting plasma sCD14 was unchanged (Table 3). Similarly, LBP was not affected by the dietary treatment over 9 weeks. The ratio of LBP:sCD14 was determined as an exploratory outcome, as recent data have indicated this may be a better marker of endotoxin exposure than either marker alone ${ }^{(18,19)}$. The $\mathrm{LBP} / \mathrm{sCD} 14$ ratio was lower in the yogurt consumption groups than in the control groups $\left(P_{\text {treatment }}=\right.$ 0.0477) (Table 3). However, plasma LPS was not affected 
by the dietary treatment. A significant dietary treatment $x$ obesity interaction effect on plasma 2-AG was detected $\left(P_{\text {treatment }} \times\right.$ obesity $\left.=0.0372\right)$. Subgroup analysis indicated yogurt consumption led to higher 2-AG in $\mathrm{YO}$ than in $\mathrm{CO}$ $(P=0 \cdot 0114)$ (Table 3$)$. On the other hand, plasma AEA was not changed.

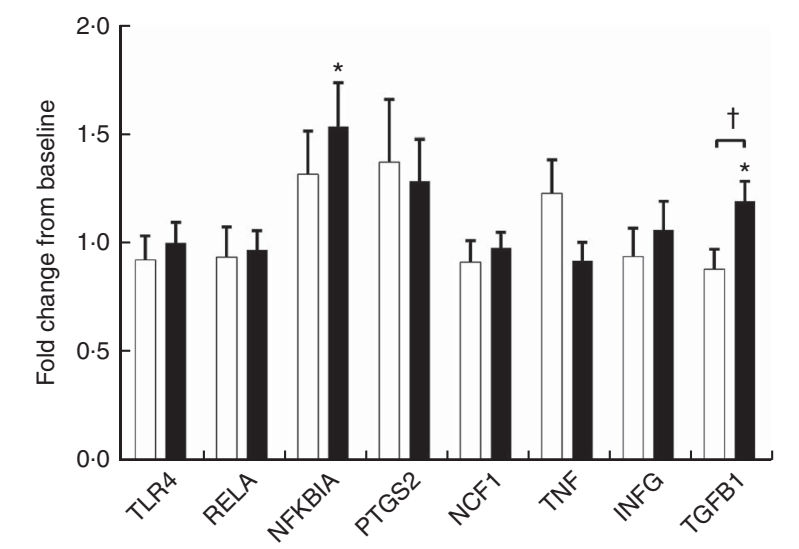

Fig. 2. Fold-change of fasting peripheral blood mononuclear cells (PBMC) gene expression between weeks 0 and 9 , during which obese participants consumed $339 \mathrm{~g}$ of yogurt or $324 \mathrm{~g}$ of a non-dairy product control snack daily (n 30/group). Values are means with their standard errors. The relative expression of target genes between groups was compared by independent $t$ test. No significant differences between yogurt obese $(\mathrm{YO}, \square)$ or control obese $(\mathrm{CO}, \square)$ gene expression was detected. The fold-change from baseline of target genes between groups was compared by independent $t$ test. The difference between weeks 0 and 9 in each group was compared by paired $t$ test. ${ }^{*} P<0.05, v$. baseline in YO. $+P<0.05$, YO v. CO. TLR4, encoding Toll-like receptor 4; RELA, encoding p65 subunit of NF-kB; NFKBIA, encoding NF-KB inhibitor alpha; PTGS2, encoding cyclo-oxygenase-2, or COX-2; NCF1, encoding the p47 subunit of NADPH oxidase; IFNG, encoding interferon- $\gamma$; TGFB1, encoding transforming growth factor $\beta 1$, or TGF $\beta$.

\section{Peripheral blood mononuclear cells mRNA expression}

Endotoxin induces the production of inflammatory cytokines via TLR4/NF- $\kappa$ B pathway. We evaluated PBMC mRNA expression of key components of the NF- $\kappa \mathrm{B}$ pathway in the obese group. At baseline, YO and $\mathrm{CO}$ gene expression did not differ (online Supplementary Fig. S1). After 9 weeks, YO NFKBIA (encoding NF- $\kappa \mathrm{B}$ inhibitor $\alpha(\mathrm{I} \kappa \mathrm{B} \alpha)$ ) and encoding transforming growth factor $\beta 1$ (TGFB1) increased by 54 and $20 \%$ from baseline, respectively (Fig. 2). In contrast, the mRNA expression of these genes did not change in CO.

\section{Anthropometric changes}

After 9 weeks intervention, BMI was affected by obesity status, but not dietary treatment $\left(P_{\text {obesity }}=0.0084\right)$ (Table 4$)$. BMI increased continuously in both YO and CO. However, dietary records did not indicate increased energy intake (online Supplementary Table S3). Despite this weight gain, YO and CO waist circumferences were unchanged. Yogurt consumption significantly reduced diastolic blood pressure $\left(P_{\text {treatment }}=\right.$ 0.0188), but not systolic blood pressure (Table 4). Within YO, diastolic blood pressure decreased by 2.82 (sEm 0.90 ) $\mathrm{mmHg}$ at week 3, with less decreases at weeks 6 and 9 .

\section{Discussion}

The present study demonstrated that consuming two servings of low-fat yogurt daily for 9 weeks reduced fasting biomarkers of chronic inflammation and endotoxin exposure in apparently healthy premenopausal women. These findings are of significance because of the known role of compromised intestinal barrier function and subsequent endotoxin exposure as a mechanism of chronic inflammation, particularly in obesity $^{(3,4)}$.

Table 4. Changes from baseline in BMI, waist circumference (WC), and blood pressure (BP) of participants during the 9-week intervention (Mean values with their standard errors; $n$ 30/group)

\begin{tabular}{|c|c|c|c|c|c|c|c|c|c|c|c|c|c|}
\hline \multirow[b]{3}{*}{ Measurements } & \multirow[b]{3}{*}{ Group } & \multicolumn{8}{|c|}{ Time (weeks) } & & & & \\
\hline & & \multicolumn{2}{|c|}{0} & \multicolumn{2}{|c|}{3} & \multicolumn{2}{|c|}{6} & \multicolumn{2}{|c|}{9} & \multicolumn{4}{|c|}{ Significance $(P)^{\star}$} \\
\hline & & Mean & SE & Mean & SE & Mean & SE & Mean & SE & Treatment & Obesity & Time & Treatment $\times$ obesity \\
\hline \multirow[t]{4}{*}{$\Delta \mathrm{BMI}\left(\mathrm{kg} / \mathrm{m}^{2}\right)$} & $\mathrm{CN}$ & 0 & 0 & 0.00 & 0.08 & $0 \cdot 10$ & 0.09 & 0.02 & 0.10 & 0.7942 & 0.0084 & 0.0130 & 0.9939 \\
\hline & YN & 0 & 0 & -0.07 & 0.06 & -0.04 & 0.09 & 0.08 & 0.11 & & & & \\
\hline & $\mathrm{CO}$ & 0 & 0 & 0.14 & 0.08 & 0.30 & 0.13 & 0.37 & 0.16 & & & & \\
\hline & YO & 0 & 0 & 0.15 & 0.08 & 0.19 & 0.09 & 0.33 & 0.11 & & & & \\
\hline \multirow[t]{4}{*}{$\Delta \mathrm{WC}(\mathrm{cm})$} & $\mathrm{CN}$ & 0 & 0 & 0.08 & 0.31 & -0.06 & 0.30 & -0.22 & 0.33 & 0.8274 & 0.9351 & 0.0956 & 0.1443 \\
\hline & YN & 0 & 0 & -0.12 & 0.13 & -0.77 & 0.33 & -0.70 & 0.32 & & & & \\
\hline & $\mathrm{CO}$ & 0 & 0 & -0.32 & 0.20 & -0.22 & 0.17 & -0.55 & 0.27 & & & & \\
\hline & YO & 0 & 0 & 0.03 & 0.16 & -0.03 & 0.36 & -0.27 & 0.43 & & & & \\
\hline \multirow{4}{*}{$\Delta$ SysBP $(\mathrm{mmHg})$} & $\mathrm{CN}$ & 0 & 0 & 0.23 & 1.48 & -1.73 & 1.04 & 0.33 & 1.57 & 0.1233 & 0.3109 & 0.4783 & 0.5015 \\
\hline & YN & 0 & 0 & 0.13 & 1.29 & -0.72 & 1.46 & -2.45 & 1.64 & & & & \\
\hline & $\mathrm{CO}$ & 0 & 0 & 2.57 & 1.22 & 1.71 & 1.39 & 1.12 & 1.70 & & & & \\
\hline & YO & 0 & 0 & $-1 \cdot 13$ & 1.48 & -0.68 & 1.29 & -0.25 & $1 \cdot 17$ & & & & \\
\hline \multirow{4}{*}{$\triangle \mathrm{DiaBP}(\mathrm{mmHg})$} & $\mathrm{CN}$ & 0 & 0 & 1.57 & 1.22 & -1.48 & 1.05 & 0.15 & 1.14 & 0.0188 & 0.2859 & 0.7587 & 0.0902 \\
\hline & YN & 0 & 0 & 0.02 & 0.77 & 0.50 & 1.09 & -0.75 & 1.11 & & & & \\
\hline & $\mathrm{CO}$ & 0 & 0 & 0.78 & 0.91 & 1.02 & 0.91 & 0.77 & 1.22 & & & & \\
\hline & YO & 0 & 0 & -2.82 & 0.90 & $-2 \cdot 10$ & 0.83 & -1.90 & 0.95 & & & & \\
\hline
\end{tabular}

$\mathrm{CN}$, control non-obese; CO, control obese; YN, yogurt non-obese; YO, yogurt obese; SysBP, systolic blood pressure; DiaBP, diastolic blood pressure.

* The effects of treatment, obesity, and treatment x obesity were determined by two-factor repeated-measures ANOVA with time as a covariate (PROC MIXED). 
Increased proinflammatory biomarkers such as IL-6, hsCRP and TNF- $\alpha$ have been associated with obesity in both adults and children $^{(20,21)}$. Consuming low-fat yogurt for 9 weeks resulted in a modest, but significant reduction of the levels of TNF- $\alpha$, which may be partly explained by reduced activation of the TLR4mediated inflammatory pathway. Upon TLR 4 activation by endotoxin, a downstream signaling cascade is triggered that leads to activation of the NF- $\kappa \mathrm{B}$ pathway ${ }^{(22)}$. I $\mathrm{I} \mathrm{B} \alpha$ (encoded by $N F K B I A$ ) inhibits the NF- $\kappa \mathrm{B}$ pathway by trapping the heterodimeric complex in the cytosol ${ }^{(23)}$. Since YO NFKBIA was increased at the end of the intervention, yogurt consumption may suppress TLR 4 activation of NF- $\kappa \mathrm{B}$. TGF- $\beta 1$ is an antiinflammatory and reparative cytokine that suppresses proinflammatory signaling from Toll-like receptors ${ }^{(24)}$. YO TGFB1 (encoding TGF- $\beta 1$ ) expression was increased by the intervention but other downstream genes of $\mathrm{NF}-\kappa \mathrm{B}$ including PTGS2, NCF1, TNF and IFNG were not affected by the intervention. Given the reduction in YO plasma TNF- $\alpha$, non-PBMC sources of TNF such as the immunocytes resident in the intestine, adipose tissue, or skeletal muscle may have contributed to this change.

Obesity is associated with subclinical endotoxaemia which increases chronic inflammation ${ }^{(5)}$. Contrary to others ${ }^{(25)}$, the obese group did not have increased LPS relative to the nonobese group in the present study. We expected plasma LPS to be reduced by yogurt consumption, however non-significant increases in plasma LPS were observed after the dietary intervention. Direct quantitation of LPS by the LAL method is challenging, due to its short half-life, low blood concentrations and the difficulty of removing interference from the blood matrix ${ }^{(26)}$. The LAL assay also does not account for lipoprotein-bound LPS $^{(27)}$. In addition, other bacterial compounds such as glycolipids and lipoproteins derived from pathogenic Gram-positive bacteria are pro-inflammatory ${ }^{(28)}$. Therefore, it is likely that quantitation of LPS by the LAL method does not account for total bioactive endotoxin, and could be masked by lipoprotein differences between experimental groups. Thus, the extent fasting LPS values in the present study reflect intestinal barrier function and true endotoxin load is unclear.

LBP and sCD14 have been proposed as surrogate biomarkers of endotoxaemia because of their roles in sequestering and translocating LPS and other bacterial compounds to inflammatory signaling pathways ${ }^{(28)}$. Similar to the present study, LBP was higher in overweight/obese individuals than in normalweight individuals, indicating low-grade chronic endotoxaemia $^{(3)}$. Serum LBP was also associated with increased abdominal obesity and proinflammatory cytokines IL- 6 and IL- ${ }^{(29)}$. However, the originally proposed primary outcome, fasting plasma sCD14, was not different between the obese and non-obese groups in the present study. Similarly, sCD14 was not associated with obesity in another study population ( $n$ 420, $55 \%$ females, age $18-92$ years $)^{(29)}$. Therefore, sCD14 alone may not be an appropriate biomarker for low-grade endotoxaemia. LBP and SCD14 act together to detoxify endotoxin. Healthy men have postprandial plasma LBP/sCD14 ratios that are correlated with plasma endotoxin ${ }^{(18,19)}$. We observed that obese participants had $27 \%$ higher plasma LBP:sCD14 ratio than the non-obese participants at baseline, which suggested increased endotoxin exposure. Moderate increases in LBP/sCD14 ratio were found in control but not the yogurt-consuming groups, suggesting protective effects of yogurt against chronic endotoxaemia.

Another surrogate biomarker of endotoxaemia is IgM Endo$\mathrm{CAb}$, which can bind to the inner core of endotoxin and protect against endotoxin ${ }^{(30)}$. In a cross-sectional study involving ninety-three age-matched middle-aged women, IgM EndoCAb in obese and obese diabetic women was 55 and $30 \%$ of nonobese participants ${ }^{(31)}$. Similarly, IgM EndoCAb level in obese participants was $69 \%$ of that in the non-obese in the present study. Yogurt consumption increased the IgM EndoCAb level in both obese and non-obese participants, suggesting decreased level of endotoxin exposure resulting from the yogurt intervention.

Intestinal barrier function is regulated in-part by eCB. 2-AG improves the intestinal barrier, whereas AEA is associated with decreased intestinal barrier function ${ }^{(4)}$. After the 9-week intervention, a modest decrease in 2-AG was observed in $\mathrm{CO}$, but not in YO. This may suggest protective effects of yogurt on intestinal barrier function relative to the control snack.

Yogurt directly increased tight junction proteins and improved barrier function in Caco-2 cells $^{(32)}$. A few dietary interventions have improved intestinal barrier function in various populations. For example, consumption of $300 \mathrm{~g} / \mathrm{d}$ yogurt containing Lactobacillus johnsonii for 4 weeks decreased plasma LBP and intestinal permeability in elderly adults with intestinal bacterial overgrowth ${ }^{(7)}$. Intervention studies on the anti-inflammatory effects of yogurt consumption are also limited. In elderly individuals, consumption of $100 \mathrm{~g} / \mathrm{d}$ yogurt containing Lactobacillus acidophilus and Bifidobacterium lactis for 2 weeks decreased faecal haptoglobin, but plasma inflammatory biomarkers were not determined ${ }^{(9)}$. In children with Helicobacter pylori, $400 \mathrm{ml} / \mathrm{d}$ yogurt containing $L$. acidophilus and $B$. lactis for 4 weeks decreased serum IL- ${ }^{(10)}$. The yogurt in the present study is a commercially available product that is more representative of typical yogurt products in the USA. Thus, probiotic fortification may not be necessary in yogurt to modulate chronic inflammation in apparently healthy women. Nevertheless, dietary intervention alone might not be sufficient to manage obesity-associated inflammation. Other strategies such as weight management and pharmaceutical approaches should be incorporated.

In this study, obese participants gained approximately $1 \mathrm{~kg}$ body weight, even though dietary records did not reveal increased energy intake. Most obese participants reported an energy intake below the calculated estimated energy requirements, indicating underreporting. The interventions supplied $54 \mathrm{~g}$ of sugar, which contributed to the increased sugar consumption by $\mathrm{YO}$ and $\mathrm{CO}$. It is estimated that individuals having higher sugar intake have $0.75 \mathrm{~kg}(95 \%$ CI $0.30,1 \cdot 19 ; P=0.001)$ more body weight than those consuming less sugar during short-term interventions ${ }^{(33)}$. Diastolic blood pressure was lower in $\mathrm{YO}$ at week 3, but rebounded at later weeks, possibly because of weight gain at later weeks. Thus, it is possible that sustained increased sugar intake diminished the beneficial effect of yogurt consumption. This study adds to the existing evidence that increased dairy product consumption might 
reduce blood pressure in obese individuals. A meta-analysis of prospective cohort studies associated an increased daily intake of $200 \mathrm{~g}$ of low-fat dairy products with decreased risk of hypertension (RR 0.96; $95 \%$ CI 0.93, 0.99) ${ }^{(34)}$.

Several limitations should be considered when interpreting the results of this study. Since only women were included as participants, the results cannot be directly extended to men. Furthermore, we did not recruit obese participants on the basis of metabolic syndrome status, which could affect response to dairy product interventions ${ }^{(35)}$. In addition, participants selfselected days to complete food records, which helps to improve participant compliance to study procedures but may introduce bias in selecting convenient days to record food intake (e.g. low diversity of foods or skipped meals). Further studies are needed to establish the extent regular yogurt consumption contributes to nutrient intake and diet quality. A strength of this study was that yogurt intervention consisted of commercially available products within the recommended dietary guidance for dairy product consumption. Notably, the yogurt was not fortified with probiotics, but this study was not designed to test the effects of fermentation. Other studies have demonstrated specific anti-inflammatory and intestinal barrier-promoting activity of probiotics ${ }^{(7,9)}$

A number of factors could have contributed to the benefits of yogurt consumption in the present study. Preclinical studies suggest milk oligosaccharides and lactoferrin promote intestinal barrier function and have anti-inflammatory properties ${ }^{(36,37)}$. Dairy product fermentation also liberates peptides with hypotensive activity, as reviewed elsewhere ${ }^{(6)}$. Other simultaneous dietary changes occurring with the intervention cannot be ruled out for their effect on biomarkers of chronic inflammation and intestinal barrier function.

In summary, this study demonstrated that consuming $339 \mathrm{~g}$ of low-fat yogurt daily for 9 weeks modestly reduced chronic inflammation and inhibited markers of endotoxaemia in apparently healthy premenopausal women. The antiinflammatory effect of yogurt consumption was partially attributable to improved intestinal barrier function indicated by EndoCAb and LBP:sCD14 in comparison to the non-dairy product control.

\section{Acknowledgements}

The authors thank Daniel Bergeron, Steven Brown, Christine Fisher, Daniel Freidenreich, Sarah Kranz, Dr Brian Kupchak, Kathryn Lainas, Eunice Mah, Beth McAvoy, Dr Stacey Mobley, Cathy Saenz, Yiming Qin, Anna Vanderleest and Liyang Xie for their technical contributions to this project.

This work was supported by a grant from the National Dairy Council. The funding agency facilitated peer review of the original research proposal, and feedback from the peer review process was considered for the final study design.

B. W. B. and R. S. B. designed the research. R. P., D. M. D., K. K. P. and D. A. M. recruited and screened participants. R. P., D. M. D., Q. G., C. C. and C. O. S. performed research and data analysis. B. W. B., R. P., R. S. B., H. M. W. and C. O. S. supervised data analysis. R. P., B. W. B. and R. S. B. were responsible for data interpretation and had primary responsibility for final content. R. P. and B. W. B. wrote the paper. All authors have approved the final manuscript.

The authors declare that there are no conflicts of interest.

\section{Supplementary material}

For supplementary material/s referred to in this article, please visit https://doi.org/10.1017/S0007114517003038

\section{References}

1. Lumeng $C N$ \& Saltiel AR (2011) Inflammatory links between obesity and metabolic disease. J Clin Invest 121, 2111-2117.

2. Gregor MF \& Hotamisligil GS (2011) Inflammatory mechanisms in obesity. Annu Rev Immunol 29, 415-445.

3. Sun L, Yu Z, Ye X, et al. (2010) A marker of endotoxemia is associated with obesity and related metabolic disorders in apparently healthy Chinese. Diabetes Care 33, 1925-1932.

4. Cani PD, Plovier H, Van Hul M, et al. (2016) Endocannabinoids - at the crossroads between the gut microbiota and host metabolism. Nat Rev Endocrinol 12, 133-143.

5. Andreasen AS, Larsen N, Pedersen-Skovsgaard T, et al. (2010) Effects of Lactobacillus acidophilus NCFM on insulin sensitivity and the systemic inflammatory response in human subjects. Br J Nutr 104, 1831-1838.

6. Pei R, Martin DA, DiMarco DM, et al. (2017) Evidence for the effects of yogurt on gut health and obesity. Crit Rev Food Sci Nutr 57, 1569-1583.

7. Schiffrin EJ, Parlesak A, Bode C, et al. (2009) Probiotic yogurt in the elderly with intestinal bacterial overgrowth: endotoxaemia and innate immune functions. Br J Nutr 101, 961-966.

8. Zeng J, Li YQ, Zuo XL, et al. (2008) Clinical trial: effect of active lactic acid bacteria on mucosal barrier function in patients with diarrhoea-predominant irritable bowel syndrome. Aliment Pharmacol Ther 28, 994-1002.

9. Matsumoto M, Ohishi H \& Benno Y (2001) Impact of LKM512 yogurt on improvement of intestinal environment of the elderly. FEMS Immunol Med Microbiol 31, 181-186.

10. Yang YJ \& Sheu BS (2012) Probiotics-containing yogurts suppress Helicobacter pylori load and modify immune response and intestinal microbiota in the Helicobacter pyloriinfected children. Helicobacter 17, 297-304.

11. Warensjö E, Jansson JH, Cederholm T, et al. (2010) Biomarkers of milk fat and the risk of myocardial infarction in men and women: a prospective, matched case-control study. Am J Clin Nutr 92, 194-202.

12. Tong X, Dong JY, Wu ZW, et al. (2011) Dairy consumption and risk of type 2 diabetes mellitus: a meta-analysis of cohort studies. Eur J Clin Nutr 65, 1027-1031.

13. Ralston RA, Lee JH, Truby H, et al. (2012) A systematic review and meta-analysis of elevated blood pressure and consumption of dairy foods. J Hum Hypertens 26, 3-13.

14. Laugerette F, Vors C, Géloën A, et al. (2011) Emulsified lipids increase endotoxemia: Possible role in early postprandial lowgrade inflammation. J Nutr Biochem 22, 53-59.

15. Campbell MJ \& Swinscow TDV (2009) Statistics at Square One, 11th ed. West Sussex: Wiley-Blackwell, Inc.

16. Pickering TG, Hall JE, Appel LJ, et al. (2005) Recommendations for blood pressure measurement in humans and experimental animals. Part 1: blood pressure measurement in humans: a statement for professionals from the subcommittee of professional and public education of the American Heart 
Association council on high blood pressure research. Hypertension 45, 142-161.

17. Thompson FE \& Subar AF (2017) Dietary Assessment Methodology. In Nutrition in the Prevention and Treatment of Disease, 4th ed., pp. 5-48 [AM Coulston, CJ Boushey, MG Ferruzzi and LM Delahanty, editors]. London: Academic Press.

18. Laugerette F, Furet JP, Debard C, et al. (2012) Oil composition of high-fat diet affects metabolic inflammation differently in connection with endotoxin receptors in mice. Am J Physiol Endocrinol Metab 302, 374-386.

19. Laugerette F, Alligier M, Bastard JP, et al. (2014) Overfeeding increases postprandial endotoxemia in men: inflammatory outcome may depend on LPS transporters LBP and sCD14. Mol Nutr Food Res 58, 1513-1518.

20. Panagiotakos DB, Pitsavos C, Yannakoulia M, et al. (2005) The implication of obesity and central fat on markers of chronic inflammation: the ATTICA study. Atherosclerosis 183, 308-315.

21. Mauras N, DelGiorno C, Kollman C, et al. (2010) Obesity without established comorbidities of the metabolic syndrome is associated with a proinflammatory and prothrombotic state, even before the onset of puberty in children. J Clin Endocrinol Metab 95, 1060-1068.

22. Manco M, Putignani L \& Bottazzo GF (2010) Gut microbiota, lipopolysaccharides, and innate immunity in the pathogenesis of obesity and cardiovascular risk. Endocr Rev 31, 817-844.

23. Scherer DC, Brockman JA, Chen ZJ, et al. (1995) Signal-induced degradation of I-kappa-B-alpha requires site-specific ubiquitination. Proc Natl Acad Sci U S A 92, 11259-11263.

24. Serhan CN \& Savill J (2005) Resolution of inflammation: the beginning programs the end. Nat Immunol 6, 1191-1197.

25. Kallio KAE, Hatonen KA, Lehto M, et al. (2015) Endotoxemia, nutrition, and cardiometabolic disorders. Acta Diabetol 52, 395-404.

26. Gnauck A, Lentle RG \& Kruger MC (2015) The Limulus Amebocyte Lysate assay may be unsuitable for detecting endotoxin in blood of healthy female subjects. $J$ Immunol Methods 416, 146-156.
27. Pais de Barros J-P, Gautier T, Sali W, et al. (2015) Quantitative lipopolysaccharide analysis using HPLC/MS/MS and its combination with the limulus amebocyte lysate assay. J Lipid Res 56, 1363-1369.

28. Schroder NWJ \& Schumann RR (2005) Non-LPS targets and actions of LPS binding protein (LBP). J Endotoxin Res 11, 237-242.

29. Gonzalez-Quintela A, Alonso M, Campos J, et al. (2013) Determinants of serum concentrations of lipopolysaccharidebinding protein (LBP) in the adult population: the role of obesity. PLOS ONE 8, e54600.

30. Poxton IR (1995) Antibodies to lipopolysaccharide. J Immunol Methods 186, 1-15.

31. Hawkesworth S, Moore SE, Fulford AJ, et al. (2013) Evidence for metabolic endotoxemia in obese and diabetic Gambian women. Nutr Diabetes 3, e83.

32. Putt K, Pei R, White W, et al. (2017) Yogurt inhibits intestinal barrier dysfunction in Caco-2 cells by increasing tight junctions. Food Funct 8, 406-414.

33. Morenga LT, Mallard S \& Mann J (2012) Dietary sugars and body weight: Systematic review and meta-analyses of randomised controlled trials and cohort studies. BMJ 345, e7492.

34. Soedamah-Muthu SS, Verberne LDM, Ding EL, et al. (2012) Dairy consumption and incidence of hypertension: a doseresponse meta-analysis of prospective cohort studies. Hypertension 60, 1131-1137.

35. Bordoni A, Danesi F, Dardevet D, et al. (2017) Dairy products and inflammation: a review of the clinical evidence. Crit Rev Food Sci Nutr 57, 2497-2525.

36. Boudry G, Hamilton MK, Chichlowski M, et al. (2017) Bovine milk oligosaccharides decrease gut permeability and improve inflammation and microbial dysbiosis in diet-induced obese mice. J Dairy Sci 100, 2471-248.

37. Anderson RC, Bassett SA, Haggarty NW, et al. (2017) Short communication: early-lactation, but not mid-lactation, bovine lactoferrin preparation increases epithelial barrier integrity of Caco-2 cell layers. J Dairy Sci 100, 886-891. 